\title{
An Ensemble of IoT with Cloud Enabled Distributed Disease Diagnosis System using Optimal Kernel Extreme Learning Machine Model
}

\author{
Z. John Bernard ${ }^{1}$, T. Vengattaraman ${ }^{2}$ \\ ${ }^{1}$ Assistant Professor, PG Department of Computer Applications, St.Joseph's College of Arts and \\ Science(Autonomous),Cuddalore, jbzbernard@ gmail.com \\ ${ }^{2}$ Department of Computer Science, Pondicherry University, Puducherry, India, vengattaramant@ gmail.com
}

\begin{abstract}
In recent times, Internet of Things (IoT) and cloud computing (CC) technologies provides diverse applications and services in the healthcare sector. In a distributed healthcare management, manyIoT devices are used to monitor the health status of the patients and send the data to the cloud server for processing. This paper devises a new IoT and cloud enabled intelligent distributed disease diagnosis model using Elephant Herd Optimization (EHO) with Kernel Extreme Learning Machine (KELM), called EHO-KELM model. The proposed EHO-KELM model initially performs the data acquisition process to collect the medical data of the patients from different sources. Then, the collected data is transmitted to the cloud server for further processing, where the EHO-KELM model gets executed to classify the patient data to identify the existence of diseases. The application of EHO algorithm helps to tune the parameters of the KELM model for better classification performance. The performance of the EHO-KELM model has been validated using two medical dataset namely diabetes and heart disease. The simulation outcome indicated that the EHO-KELM model has demonstrated effective outcome with the maximum classification accuracy of $94.25 \%$ on the applied heart disease respectively.
\end{abstract}

Key words: IoT, Cloud computing, Distributed system, Disease diagnosis, Healthcare

\section{INTRODUCTION}

Nowadays, Internet of Things (IoT) becomes a more interesting topic among the researchers, public area, and industries. While conventional internet helps communication among limited device count and individuals, it relates all type of linked "Things" into a broad web associated with computing intelligence without individual's involvement. The acceptance of IoT and the growth of wireless communication models permit sending patient's health status to caretaker in real time. On the other hand, distributed computing in healthcare comprises a set of IoT based medical gadgets and networking devices, which transmit and interact data with one another for passing the patient data to the cloud server. Moreover, various existing sensor and the convenient device could evaluate particular person's physiological limit such as Respiration Rate (RR) Blood Pressure (BP) and Heart Rate (HR) in just single tap. Still, it is in the initial stage of development, business and industrial sectors have rapidly supported the control of IoT in their active system, and it observed development in the invention includes user familiarity. CC brings IT services including data analytics, software, networking, databases, and servers over the web to supply more rapidly operations, responsive income, and economies of scale. In addition to this, the existing transition from centralized theory of CC to decentralized theory (fog computing) are holding the topmost parts. Fog computing executes data analysis on edge devices, so it permits real time processing, develops privacy of the data, and it minimizes the costs too. The growth of CC, Artificial Intelligence (AI), and portable devices certifies a definite groundwork for the development of IoT in the medicinal sector to modernize all features of individual lives. Figure 1 shows the structure of IoT with cloud based healthcare method.

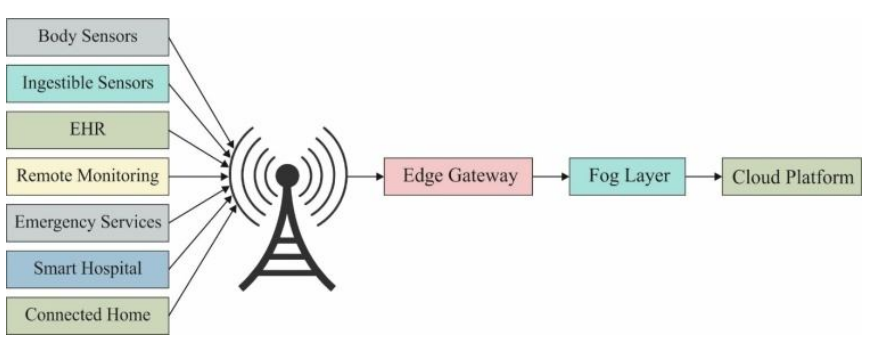

Figure 1: Structure of IoT with Cloud based Healthcare Model

IoT gives proper solution for different applications that includes all aspect of growth like health care, smart traffic management, supply chain, structural health monitoring, emergency services, retail, smart cities, industrial management, security, and waste management. Based on details by CISCO, at the end of 2030, 500 billion devices 
would be connected, which is nearly equal to fifty-eight smart devices per human being universally. By Dec 2017, IoT market research approved by Statistic shown that global IoT market worth will attain 8.9 trillion USD by 2020 , and seven percentage of the overall market worth come from the health care field. The combination process of IoT and CC into the health care field assists the medical professionals to give rapid and efficient health care services for better patient experience. As a result, it brings better health care services, patient familiarity, and less paper work for medical professionals. The IoT in health care framework (IoTHeF) is examined as a primary feature of IoT in health care since it aids health care application for complete usage of IoT and CC. The framework provides protocol for maintaining the communication and broadcast of raw medicinal signal from a variety of sensors and smart devices to a web of fog nodes. Given in figure 2, there are 3 important modules of IoTHeF, which consist of platform, structure, and topology. Every module serves a definite role in the IoT health care frame. The system could assemble information about status of the patient via various sensors. Later, the collected information was passed to the remote server for diagnosing, and the outcomes were viewed in actual time.

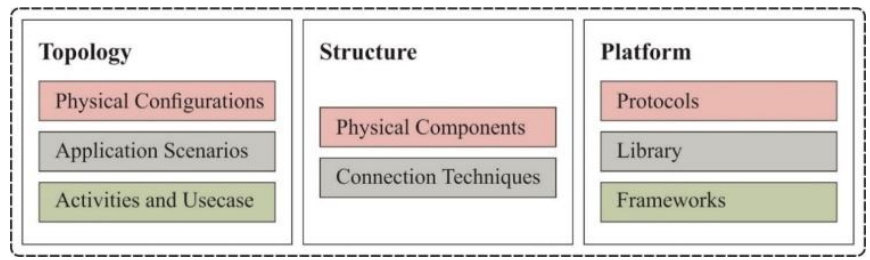

Figure 2: Components and Functions of IoT Healthcare

In past decades, several computations are carried out by the developers. [1] implied an efficient Cyber Physical System to support the multi-sites as well as multi-products development. [2] applied a new system for car camera surveillance that employs mobile CC approach for Deep Learning (DL). It examines the objects present in the saved videos that have been captured while driving the car and select specific portions of a video which has to be stored privately in CC. It is operated on the basis of training phase, recognition phase as well as data collection phase. An optimal prediction value can be accomplished from this approach.

[3] presented an online health tracking mechanism named as Healthcare Industrial IoT. It is applicable for detecting the patients' health details and prevents from fatal rate. The gathered patient details are examined with the application of sensors and clinical tools. The main objective of this method is to eliminate the medical errors and different identity thefts using security measures like watermarking and signal extensions. [4] projected a user based sensing approach for elderly and disabled people. The primary goal of this approach is offering service based on the emergency situations like major accidents, heart attack, and pregnancy and so on. [5] provided smart and collective security techniques for limiting the risks in an IoT centric healthcare platform. Furthermore, specific emphasis is provided to review the state-of-art network environment, applications and industrial improvement inIoT based medical solutions. [6] used an intelligent medical analyzing method termed as neuro-fuzzy temporal knowledge representation used to detect and diagnose diverse types of deadly disease.

This paper introduces an effective IoT and cloud enabled intelligent distributed disease diagnosis model using Elephant Herd Optimization (EHO) with Kernel Extreme Learning Machine (KELM), called EHO-KELM model. The proposed EHO-KELM model involves two major processes namely data acquisition and data classification. The data acquisition process involves collecting the medical data of the patients from different sources. Then, the collected data is transmitted to the cloud server for further processing, where the EHO-KELM model gets executed to classify the patient data to identify the existence of diseases. A detailed set of experimental validation is carried out and the results are analyzed under diverse aspects.

\section{THE PROPOSED EHO-KELM MODEL}

The working principle of the proposed EHO-KELM model is depicted in figure 3 . The figure showed that the proposed EHO-KELM model has initially performed data acquisition process where the data gets gathered from three different sources namely electronic health records (EHRs), medical data using IoT sensors attached to human body and UCI repository data. Then, the collected data gets transmitted to the cloud processing system where the data preprocessing and classification processes will be carried out. The preprocessing step eliminates the unwanted data and improved the data quality. Finally, EHO-KELM algorithm is applied as a classification model to identify the presence of the disease in the healthcare data. The classified output will be sent to the alert system, which incorporates hospital database, physicians, and patients to get aware of the medical output. The processes involved in this model are discussed in the following subsections.

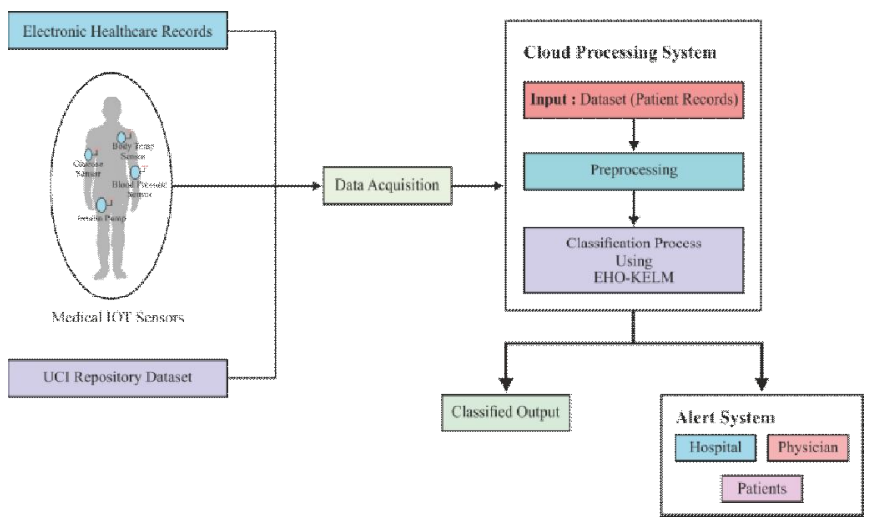

Figure 3: Architecture of the Enhanced Fuzzy Resolution Mechanism using ANFIS 


\subsection{Data acquisition and Preprocessing}

At the beginning stage, the data is collected using the IoT gadgets connected to the human, EHR, and data repository. This study mainly focused on the detection of diabetes and heart disease. The IoT devices placed on the human body collects electrocardiogram (ECG), heart rate, blood pressure, peripheral pulse oximetry (SpO2) level, glucose level, blood fat level, and pulse rate information for the detection of heart disease. Besides, glucose sensor, blood pressure and pulse sensor are used to collect the data regarding diabetes disease diagnosis. When the data is gathered, it gets transmitted to the cloud processing unit. In addition, when the data is available at the cloud, preprocessing is carried out. Firstly, data transformation procedure is done to convert the data from categorical to numerical values. Second, normalization of data is performed to transform the data into a uniform range.

\subsection{EHO-KELM Model}

In this section, the processes involved in the EHO algorithm, KELM and hybridization of EHO-KELM has been discussed in detail.

\section{A. EHO algorithm}

EHO method is defined as swarm intelligence (SI) mechanism. It is a meta-heuristic searching model that is evolved from herding behavior of elephants. The specific behavior is defined in the following. The population of elephants is composed of subgroups, named as clans that are constrained with many maximum elephants. Every clan contains a leadership named as matriarch, whereas male elephants (ME) leave the group once it has grown into adult and lives solely [7]. With respect to EHO, such behaviours are classified into 2 phases namely,

- Clan update that upgrades the recent position of elephants and matriarch

- A separation that improves the population diversity at later search phase

\section{B. Structure of KELM}

Recently, KELM is defined as novel learning method. It is evolved from ELM, which ensures in producing resourceful generalization in a large number of real-time applications. In depth, a short definition of KELM is provided in this approach, where the information is acquired from ELM was projected for single-hidden layer feed forward neural networks (FFNN) which is named to be generalized SLFNs with no tuning parameters in the hidden layer. The 2 significant metrics which are provided in RBF kernel as penalty parameter $\mathrm{C}$ as well as kernel parameter gamma $\gamma[8]$

\section{Parameter Optimization of KELM using EHO algorithm}

It is apparent that the KELM is influenced mainly by two variables namely $\mathrm{C}$ and $\gamma$. Here, EHO algorithm [9] is applied for the identification of these two variables and derived
EHO-KELM algorithm. The working of EHO-KELM is mainly based on two processes namely parameter optimization and classification property assessment. At the time of optimizing parameters, 10 -fold cross validation [10-12] is employed on the entire dataset. Once the process of optimizing inner parameters are completed, the optimal parameters are given as input to the KELM model for performing the classification process to predict the presence of disease. The classifier accuracy is considered in the design of fitness function, as given below:

$$
\begin{aligned}
\text { Test }_{\text {Accuracy }}= & \text { Average }_{\text {Accuracy }} \\
& =\frac{\sum_{i=1}^{10} \text { Test }_{\text {Accuracy }}(i)}{10}(1)
\end{aligned}
$$

\section{PERFORMANCE VALIDATION}

An extensive set of experimental analysis is carried out to ensure the effective performance of the EHO-KELM model on the applied heart disease dataset.Table 1 and figure 4 showcase the comparative analysis of the presented EHO-KEM method for HD prediction with respect to sensitivity, specificity and accuracy. The figure stated that the RT model is said to be inferior performer, which has reached minimum value of sensitivity, specificity and accuracy of $74.40 \%, 71.11 \%$ and $72.93 \%$ correspondingly. Concurrently, the MLP method has provided to attain gradual than SVM model, but not than alternate models with sensitivity, specificity and accuracy values of $78.40 \%, 79.52 \%$ and $78.87 \%$ correspondingly.

Table 1: Result Analysis of Existing with Proposed EHO-KELM Method for Heart Disease Prediction

\begin{tabular}{|l|c|c|c|}
\hline Methods & Sensitivity & Specificity & Accuracy \\
\hline EHO-KELM & 93.56 & 94.67 & 94.25 \\
\hline LR & 83.81 & 85.38 & 84.48 \\
\hline MLP & 78.40 & 79.52 & 78.87 \\
\hline RBFNetwork & 85.18 & 81.56 & 83.49 \\
\hline RF & 81.97 & 82.44 & 82.17 \\
\hline RT & 74.40 & 71.11 & 72.93 \\
\hline
\end{tabular}

In line with this, the RF model has performed well than existing models and exhibited reasonable sensitivity, specificity and accuracy values of $81.97 \%, 82.44 \%$ and $82.17 \%$ respectively. Simultaneously, the LR model has implied somewhat better results over the RF with the appreciable sensitivity, specificity and accuracy values of $83.81 \%, 85.38 \%$ and $84.48 \%$ correspondingly. Then, the RBFNetwork model has showcased competing performance with the closer sensitivity, specificity and accuracy values of $85.18 \%, 81.56 \%$ and $83.49 \%$ respectively. Thus, the proposed EHO-KELM model has accomplished maximum classification performance that is ensured from the higher 
sensitivity, specificity and accuracy values of $93.56 \%$, $94.67 \%$ and $94.25 \%$ respectively.

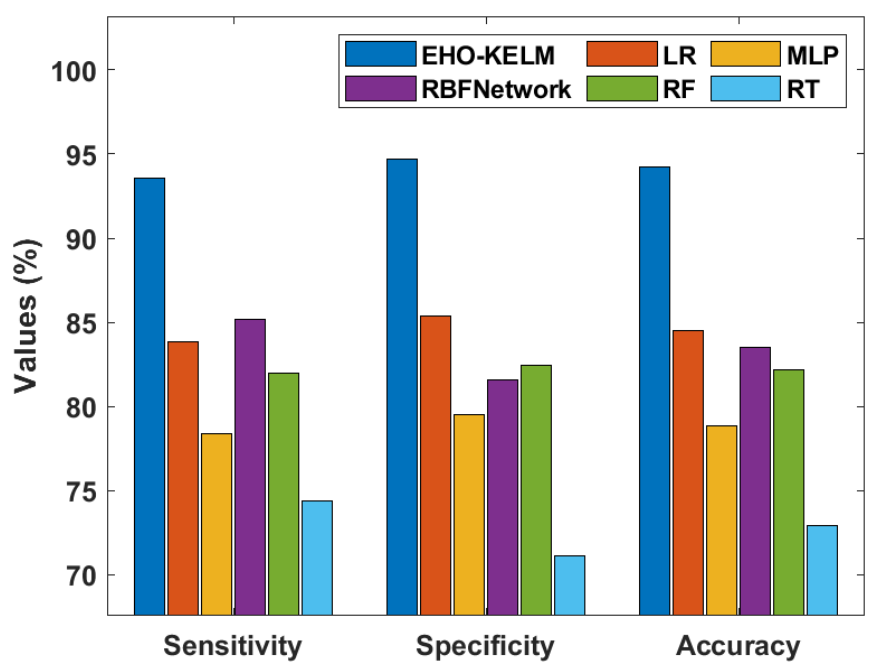

Figure 4: Comparative analysis ofEHO-KEM model for Heart Disease Prediction

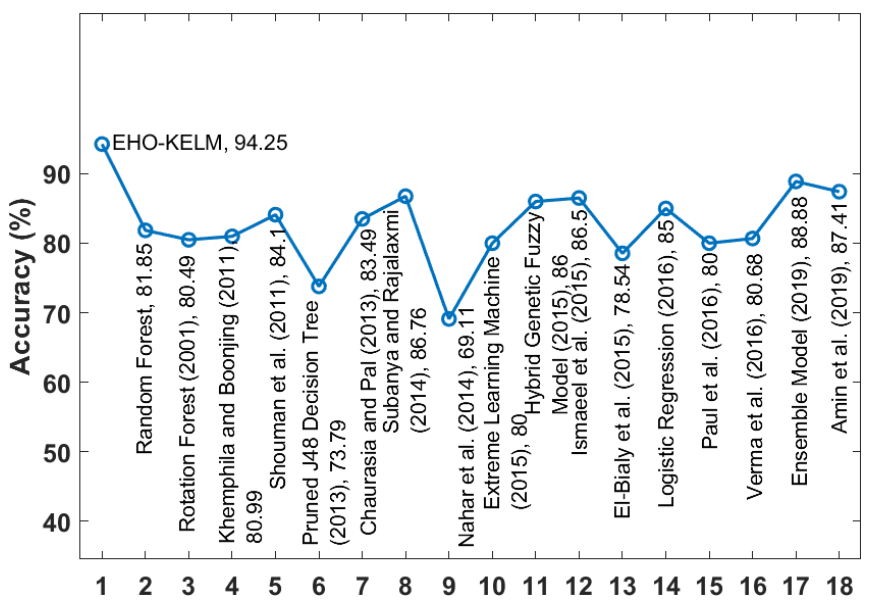

Figure 5: Comparison with Recent Methods in terms of Accuracy for Heart Disease Prediction

For validating the superior results of the proposed EHO-KELM model on the applied heart disease dataset, a comparison of the results attained by the EHO-KELM model has been made with the recently proposed models as shown in figure 5. The EHO-KELM model has reached to a maximum classification performance by attaining a higher accuracy of $94.25 \%$. The abovementioned experimental validation ensured that the EHO-KELM model has outperformed all the compared methods under diverse aspects. Therefore, it can be employed as an effective IoT and cloud enabled prediction tool for disease diagnosis.

The detailed simulation analysis ensured that the EHO-KELM model has performed well on both diabetes and heart disease dataset. The experimental outcome stated that the EHO-KELM model can be employed as an effective decision making tool for assisting physicians in real time.

\section{CONCLUSION}

This paper has introduced an effective IoT and cloud enabled intelligent distributed disease diagnosis model using EHO-KELM model. The EHO-KELM model involves three modules namely data acquisition, cloud processing system and alert system. The data acquisition process involves collecting the medical data of the patients from different sources. Then, the collected data is transmitted to the cloud server to perform classification process and identify the presence of the disease in the healthcare data. The classified output will be sent to the alert system, which incorporates hospital database, physicians, and patients to get aware of the medical output. The performance of the EHO-KELM model has been validated using two medical dataset namely diabetes and heart disease. The simulation outcome indicated that the EHO-KELM model has demonstrated effective outcome with the maximum classification accuracy of $94.25 \%$ on the applied heart disease respectively. In future, the lightweight cryptographic algorithms can be incorporated in the IoT devices for secure data transmission to the cloud server.

\section{REFERENCES}

1. Hyunsoo Lee, Framework and development of fault detection classification usingIoT device and cloud environment, J. Manuf. Syst. 43 (2017) 257-270.

2. Chien-Hung Chen, Che-Rung Lee, Walter Chen-HuaLu, Smart in-car camera system using mobile cloud computing framework for deep learning, Veh. Commun. 10 (2017) 84-90.

3. M.S. Hossain, G. Muhammad, Cloud-assisted industrial internet of things (IIoT)-enabled framework for health monitoring, Comput. Netw. 101 (2016) 192-202.

4. A. Hussain, R. Wenbi, A. Lopes, M. Nadher, M. Mudhish, Health and emergency care platform for the elderly and disabled people in the smart city, J. Syst. Softw. 110 (2015) 253-263.

5. S.M.R. Islam, D. Kwak, H. Kabir, The internet of things for health care: A comprehensive survey, IEEE Access 3 (2015) 678-708.

6. R. Sethukkarasi, S. Ganapathy, P. Yogesh, A. Kannan, An intelligent neuro fuzzy temporal knowledge representation model for mining temporal patterns, J. Intell. Fuzzy Syst. 26 (3) (2014) 1167-1178.

7. Wang, G.G., Deb, S. and Coelho, L.D.S., 2015, December. Elephant herding optimization. In 2015 3rd International Symposium on Computational and Business Intelligence (ISCBI) (pp. 1-5). IEEE.

8. Wang, M., Chen, H., Li, H., Cai, Z., Zhao, X., Tong, C., $\mathrm{Li}$, J. and $\mathrm{Xu}, \mathrm{X} ., 2$ 2017. Grey wolf optimization evolving kernel extreme learning machine: Application to bankruptcy prediction. Engineering Applications of Artificial Intelligence, 63, pp.54-68.

9. Pradhan, M., Roy, P.K. and Pal, T., 2018. Oppositional based grey wolf optimization algorithm for economic dispatch problem of power system. Ain Shams Engineering Journal, 9(4), pp.2015-2025. 
Z. John Bernard et al., International Journal of Emerging Trends in Engineering Research, 8(10), October 2020, 7151 - 7155

10. Chandra Sekhar Reddy, N. Purna Chandra Rao Vemuri, Govardhan, A. An Emperical Study on Support Vector Machines for Intrusion Detection, International Journal of Emerging Trends in Engineering Research, Vol. 7, No. 10, pp. 383-387, October 2019.

11. Rufo I. Marasigan Jr, Alvin SarragaAlon, Mon Arjay F. Malbog, Joshua S. Gulmatico, Copra Meat Classification using Convolutional Neural Network, International Journal of Emerging Trends in Engineering Research, Vol. 8. No. 2, February 2020

12. Subramaniyam, S, Performance Analysis on Diesel Engine using Neem and Soya Bean Oil,International Journal of Emerging Technologies in Engineering Research (IJETER), Vol. 5, Issue 8, August 2017. 